\author{
ДАНИЛОВА Н.Е., Северо-Кавказский федеральный университет \\ CEMEHOBA Ю.А., г. Ставрополь, Россия \\ СМЕРЕК Ю.Л., \\ ЗАКИНЯН Р.Г. \\ zakinyan@mail.ru
}

\title{
ВЛИЯНИЕ ПОДОБЛАЧНОЙ КОНВЕКЦИИ НА РАЗВИТИЕ ОБЛАЧНОЙ КОНВЕКЦИИ
}

Введение:

Облачная конвекция является причиной опасных явлений погоды [23]. Так сильные дожди и последующие наводнения являются прямым следствием конвективных штормов. Явленияшторма часто сильно локализованы, поэтому прогнозирование того, почему, где и когда шторм развивается, является одной из самых сложных проблем в прогнозе погоды [10]. На сегодняшний день оперативные численные модели часто не могут предсказать местоположение и время начала облачной конвекции при инициировании ее процессамив подоблачном слое, поскольку эти процессы недостаточно хорошо представлены в моделях. Однако влияние этих процессов на инициирование облачной конвекции часто отмечается в исследованиях $[25,39]$, так что для прогнозирования начала облачной конвекции требуется знание термодинамики и динамикиподоблачного слоя.

Материалы и методы: Для прогноза параметров облачной конвекции используется данные радиозондирования, которые наносятся на аэрологическую диаграмму. Как правило, при этом делается допущение, что скорость восходящих потоков и перегрев на уровне конденсации равны нулю. Но это не всегда так. Поэтому представляется важным определить, как параметры приземного слоя влияют на

Результаты исследования:

Обсуждение и заключение:

Ключевые слова: значения параметров облачной конвекции на уровне конденсации.

в статье разработана математическая модель конвекции влажного подоблачного слоя атмосферы. Математическая модель представляет собой систему уравнений движений, теплопроводности и неразрывности, записанные в двумерной вертикальной плоскости. Получено аналитическое решение указанной системы. Установлены выражения для уровня конвекции, уровня максимальной скорости, для профиля вертикальной и горизонтальной составляющей скорости, вертикальное распределение температуры. Из указанных выражений выведен критерий развития подоблачной конвекции. Полученные выражения позволяют в свою очередь вывести выражения для параметров конвекции на уровне конденсации. А это в свою очередь позволяет вывести критерий развития облачной конвекции. Установлено, что развитие облачной конвекции зависит, как от абсолютных значений дефицита точки росы в приземном слое, так и в большей степени от вертикального градиента массовой доли водяного пара. В частности при критическом значении вертикального градиента массовой доли водяного пара наблюдается (взрывной рост) конвекции.

Получены аналитические выражения для дефицитов точки росы в приземном слое, определяющие значения функции перегрева и скорости восходящих потоков на уровне конденсации. Показано, что на развитие подоблачной конвекции существенное влияние оказывает значение массовой доли водяного пара. Установлено, что при значениях градиента массовой доли водяного пара, приближающихся к критическому значению, наблюдается «взрывной» рост конвективных ячеек в подоблачном слое. Также показано, что существует критическое значение градиента массовой доли водяного, способствующее развитию облачной конвекции.

приземный слой атмосферы, подоблачная конвекция, уровень конденсации облачная конвекция, двумерная модель конвекции, аналитическое решение/ 
Danilova N.Ye., North-Caucasian Federal University

Semyonova Yu.A., Stavropol, Russia,

SmerekYu. L, Zakinyan R.G.

zakinyan@mail.ru

\section{Influence under-cloudy convections on development of a cloudy convection}

Introduction:

Cloud convection causes hazardous weather [23]. So heavy rains and subsequent floods are a direct consequence of convective storms. Storm phenomena are often highly localized, so predicting why, where and when a storm develops is one of the most difficult problems in weather forecasting [10]. Today, operational numerical models often cannot predict the location and time of the onset of cloud convection when it is initiated in the skid layer, since these processes are not well represented in the models. However, the influence of these processes on the initiation of cloud convection is often noted in studies $[25,39]$, so that to predict the beginning of cloud convection requires knowledge of the thermodynamics and dynamics of the sub-cloud layer.

Materials and methods: To predict the parameters of cloud convection, radiosonde data is used, which are plotted on the upper-air diagram. As a rule, it is assumed that the rate of ascending flows and overheating at the level of condensation are zero. But it's not always the case Therefore, it is important to determine how the parameters of the surface layer affect the values of the parameters of cloud convection at the level of condensation.

Results: In the article the mathematical model of a convection moist under-cloud layer atmosphere is developed. The mathematical model represents system of the equations of movements, heat conductivity and the continuity, recorded in a two-dimensional vertical plane The analytical solution of the indicated system is received. Expressions for level of convection, level of the maximum velocity, for vertical and horizontal components ofvelocity, vertical distribution of temperature are established. From the indicated expressions the criterion of development under cloudy convections is derived. The received expressions allow derived in turn expressions for convection parameters on a condensation level. And it in turn allows derived criterion of development of a cloudy convection. It is established that development of a cloudy convection depends, as from absolute values of deficit of dew point in a ground layer, and in a greater degree from a vertical gradient of a mass fraction of a water vapor. In particular at critical value of a vertical gradient of a mass fraction of water vapor «explosive growth» convections is observed.

Discussion and conclusion

Analytical expressions are obtained for the dew point deficiencies in the surface layer which determine the values of the overheating function and the ascending flow rate at the condensation level. It has been shown that the development of subliminal convection is significantly affected by the value of the mass fraction of water vapor. It has been established that with the values of the gradient of the mass fraction of water vapor approaching the critical value, there is an "explosive" growth of convective cells in the sublayer. It is also shown that there is a critical value of the gradient of the mass fraction of water, contributing to the development of cloud convection.

Keywords: $\quad$ surface layer of atmosphere, under-cloudy convection, a condensation level, a cloudy convection, two-dimensional model of convection, the analytical solution

\section{Введение}

Очевидно, что характеристики приземного слоя влияют на развитие конвекции в подоблачном слое, что в свою очередь сказывается на развитии облачной конвекции. Вопрос заключается в том, какие характеристики приземного слоя в большей или меньшей степени влияют на развитие, в конечном счете, облачной конвекции. Так, в статье [11] приводятся экспериментальные подтверждения влияния вертикального профиля влажности на развитие конвекшии. Исследованию влияния вертикального распределения 
температуры и влажности приземного слоя на состояние свободной тропосферы посвящена статья [33]

Надо заметить, что, хотя конвективное движение является одним из самых распространенных видов движений в атмосфере, как физика этого процесса, так и его математическое описание остаются открытыми. Считается, что конвекция начинается с формированием термиков [14]. Физика образования термиков аналогична фазовому переходу. Здесь аналогично при определенных условиях возникает термик критического размера. Если размер термика меньше критического, то он неустойчив и со временем исчезает. Если же размер термика больше критического размера, то он устойчив. Далее он отрывается и растет. Вопрос о размере критического термика с точки зрения теории остается открытым. Как правило, его определяют из баланса силы плавучести и силы сопротивления Стокса.

Есть еще один аспект, который делает представленное в настоящей работе исследование актуальным. В настоящее время эффекты, связанные с мелкомасштабными процессами, к которым относится мелкая конвекция параметризуются в обшей циркуляционной, региональной или мезомасштабной моделях. От того, насколько хорошо мы понимаем с точки зрения физики и умеем описывать эти процессы, будет зависеть качество прогнозов в глобальных и региональных моделях $[17,28]$. Подоблачная конвекция определяет вертикальное распределение влажности, теплоты и импульсаи играет ключевуюроль в глобальном гидрологическом и энергетическом цикле $[2,7,16$, $24,34,41]$. Для углубления понимания роли процессов подоблачного слоя в климатических моделях использовались простые одномерные модели $[3,3,8$, $12,15,26,33]$ и их совершенствование является актуальной задачей.

Облачная конвекция является причиной опасных явлений погоды [23]. Так сильные дожди и последующие наводнения являются прямым следствием конвективных штормов. Явленияшторма часто сильно локализованы, поэтому прогнозирование того, почему, где и когда шторм развивается, является одной из самых сложных проблем в прогнозе погоды [10]. На сегодняшний день оперативные численные модели часто не могут предсказать местоположение и время начала облачной конвекции при инициировании ее процессамив подоблачном слое, посколыку эти процессы недостаточно хорошо представлены в моделях. Однако влияние этих процессов на инициирование облачной конвекции часто отмечается в исследованиях [25, 39], так что для прогнозирования начала облачной конвекции требуется знание термодинамики и динамикиподоблачного слоя.

Проведенные ранее полевые исследования дают представление о процессах, вызывающих инициирование облачной конвекции $[18,35,37,39]$. Хотя имеется хорошее понимание общей динамики рассматриваемых процессов, начальная стадия развития конвективньх ячеек остается недостаточно изученной [4] 
В ряде исследований предполагалось $[9,11]$, что инициирование облачной конвекции очень чувствительно к вариациям температуры и влажности в подоблачном слое.В частности, поле влажности подоблачного слоя является ключевым фактором для понимания инициирования осадкообразующей конвекции [20]. Небольшие изменения в массовой доле водяного пара могут управлять процессом и определять, происходят ли штормы или нет. И что особенно важно, изменения приблизительно в $1 \mathrm{~g} / \mathrm{kg}$ в массовой доле водяного пара могут дать диаметрально противоположные прогнозы:отсутствие конвекции или интенсивная конвекция [11]. Много исследований проведено по изучению влияния влажности поверхности на развитие конвекции $[31,44]$ Главный механизм положительной обратной связи заключается в том, что влажный грунт обогащает воздух пограничного слоя влажностью $[5,13]$.

Таким образом, понимание и успешное предсказание штормового инициирования зависят от точногоопределенияначальныхтермодинамических и кинематических полей подоблачного слоя, с особым вниманием к полям влажности водяного пара. Вообще, типичное разрешение обычных систем наблюдения, таких как радиозонды, недостаточно для того, чтобы наблюдать изменчивость параметров в подоблачном слое атмосферы. В ряде исследований [37] предполагалось, что критическим фактором, ограничивающим прогноз конвективных осадков, является неопределенность измерения вертикального распространения водяного пара. Радиозонды, являющиеся традиционными средствами измерения содержания водяного пара, недостаточны для этих целей. Это связано с тем, что они предоставляют информацию о вертикальном профиле в широко распределенных пунктах зондирования и обычно доступны только два раза в день, иногда содержа существенные погрешности $[32,36]$.

Таким образом, влиянию характеристик приземного слоя атмосферы на развитие, как крупномасштабной циркуляции $[1,27]$, так и развитие облачной конвекции посвящено много работ [6]

В настоящей работе и подоблачная и облачная конвекция описываются с помощью двухмерной модели. Находятся соответствующие аналитические решения, а также выявляются критерии влияния параметров приземного слоя атмосферы на развитие облачной конвекции.

\section{Материалы и методы исследования}

Для прогноза параметров облачной конвекции используется данные радиозондирования, которые наносятся на аэрологическую диаграмму. Как правило, при этом делается допущение, что скорость восходящих потоков и перегрев на уровне конденсации равны нулю. А это не всегда так. Поэтому представляется важным определить, как параметры приземного слоя влияют на значения параметров облачной конвекции на уровне конденсации. 
В работе [42] было получено аналитическое решение двумерной задачи тепловой конвекции сухого воздуха в атмосфере. Причем воздух считался абсолютно сухим. А в работе [43] получено аналитическое решение двумерной модели конвекции влажногонасыщенного воздуха. Последний случай для краткости изложения будем называть «облачной» конвекцией. Таким образом, у нас есть аналитическое решение двумерной модели конвекции сухого воздуха, которую будем называть «подоблачной» конвекцией, и аналитическое решение «облачной» конвекции. Целью настоящей работы является применить полученныс в работах $[42,43]$ результаты для анализа влияния характеристик приземного слоя атмосферы на развитие облачной конвекции.

Для описания облачной конвекции нужно знать параметры конвекции, такие как функция перегрева, скорость восходящих потоков и массовая доля водяного пара на уровне конденсации. Эти параметры могут быть найдены из рассмотрения подоблачной конвекции. Она в свою очередь определяется характеристиками приземного слоя. Таким образом, характеристики приземного слоя определяют характер облачной конвекции. Задача настоящей работы состоит в том, чтобы эту связь установить. Ранее, как было отмечено во введении, такие исследования выполнялись численно и экспериментально или же использовались одномерные модели конвекции. Здесь же мы исследуем данную задачу аналитически в рамках двумерной нелинейной модели конвекции. Данный анализ, как уже было сказано, основан на полученных нами ранее аналитических решениях конвекции сухого и влажного насыщенного воздуха.

\section{Уравнения тепловой конвекции влажного воздуха}

Подоблачный воздух не является сухим. Поэтому нам необходимо, предварительно, описать конвекцию влажного ненасыщенного воздуха. По аналогии с работой [42], где мы получили аналитическое решение двумерной модели конвекциисухого воздуха,получим теперь решение для конвекции влажного ненасыщенного воздуха и рассмотрим влияние параметров приземного слоя атмосферы на развитие облачной конвекции, т.е. на значения параметров конвекции на уровне конденсации.

В настоящем разделе мы шаг за шагом повторим вывод, проделанный нами в работе [42], но учтем, что воздух теперь влажный. Уравнение движения рассматривается в плоскости $(x, z)$. Давление представляется в виде суммы давлений в состоянии статики и его возмущения: $p=\bar{p}+p^{\prime}$. Плотность влажного воздуха определим выражением:

$$
\rho_{\mathrm{i}}=\rho_{\mathrm{e}}(1-\alpha \Delta T-\beta \Delta s)
$$

где $\quad \alpha=\frac{1}{T_{0}}-\quad$ коэффициент теплового расширения воздуха $\left(\mathrm{T}_{0}=273 \mathrm{~K}\right)$;

$$
\Delta T(z)=T_{\mathrm{i}}(z)-T_{\mathrm{e}}(z)-\text { функция перегрева; }
$$


$\Delta T_{i}, T_{e}-$ температура воздуха, соответственно, возмущенной и невозмушенной атмосферы;

$S_{-} \quad$ массовая доля водяного пара;

$\Delta s=s_{\mathrm{i}}-s_{\mathrm{e}}-$ функция пересыщения;

$\beta \equiv \frac{M_{\mathrm{d}}}{M_{\mathrm{v}}}-1=0.608$;

$M_{\mathrm{d}}=29 \mathrm{~g} / \mathrm{mol}-$ молярная масса сухого воздуха;

$M_{\mathrm{v}}=18 \mathrm{~g} / \mathrm{mol}$ - молярная масса водяного пара.

Здесь $\rho_{i}-\quad$ плотность воздуха возмущенной атмосферы;

$\rho_{\mathrm{e}}-\quad$ плотность воздуха в состоянии статикиатмосферы. Параметры окружаюшей атмосферы мы рассматриваем как невозмушенное состояние. Для атмосферы $\alpha \Delta T<1$. Считается, что подъем воздушной частицы происходит адиабатически. Другими словами, при приближении (1) предполагается, что плотность воздуха зависит от температуры и массовой доли водяного пара и не зависит от давления [14, 21].

Функция перегрева равна:

$$
\Delta T(z)=\Delta_{0} T-\Delta \gamma \cdot z
$$

где $\Delta_{0} T=T_{\mathrm{i} 0}-T_{\mathrm{e} 0}-$ значение функции перегрева вблизи поверхности земли;

$\Delta \gamma=\gamma_{\mathrm{a}}-\gamma$, где $\gamma-$ градиент температуры воздуха невозмущенной атмосферы в состоянии статики;

$\gamma_{\mathrm{a}}-\quad$ сухоадиабатический градиент температуры;

$T_{10}$ - температура воздуха вблизи поверхности земли возмущенной атмосферы;

$T_{\mathrm{e} 0}-\quad$ температура воздуха вблизи поверхности земли невозмущенной атмосферы в состоянии статики.

Для адиабатически поднимающейся воздушной частицы массовая доля водяного пара остается постоянной $s_{\mathrm{i}}(z)=s_{\mathrm{i} 0}$, где $s_{\mathrm{i} 0}-$ массовая доля водяного пара вблизи поверхности земли. Пусть в невозмущенной атмосфере в состоянии статики массовая доля водяного пара убывает по линейному закону

$$
s_{\mathrm{e}}(z)=s_{\mathrm{e} 0}-b z
$$

где $b-$

градиент массовой доли водяного пара. Тогда для функции пересыщения получим выражение

$$
\Delta s(z)=\Delta_{0} s+b z
$$

$$
\Delta_{0} s=s_{\mathrm{i} 0}-s_{\mathrm{e} 0}
$$


- пересыщение у поверхности земли. Выражение для плотности воздушной частицы запишется в виде

$\rho_{\mathrm{i}}=\rho_{\mathrm{e}}\left[1-\left(\alpha \Delta_{0} T+\beta \Delta_{0} s\right)+(\alpha \Delta \gamma-\beta b) z\right]$.

Отсюда найдем уровень выравнивания плотностей воздуха в воздушной частице и невозмущенной атмосфере

$z_{\rho}=\frac{\alpha \Delta_{0} T+\beta \Delta_{0} s}{\alpha \Delta \gamma-\beta b}$.

Из формулы (6), формально принимая $\beta=0$, получим выражение для уровня выравнивания температур $z_{\mathrm{t}}=\frac{\Delta_{0} T}{\Delta \gamma}$, которое следует непосредственно из формулы (2). Отсюда видно, что уровень вьравнивания плотностей воздуха для влажной частицы выше, чем уровень выравнивания температур.

Принимая во внимание уравнение статики, которое имеет место для невозмущенной атмосферы, система уравнений, описывающих тепловую конвекцию влажного ненасыщенного воздуха в стационарном состоянии, представится в виде:

$$
\begin{aligned}
& u \frac{\partial u}{\partial x}+w \frac{\partial u}{\partial z}=-\frac{1}{\rho_{\mathrm{e}}} \frac{\partial p^{\prime}}{\partial x}, \\
& u \frac{\partial w}{\partial x}+w \frac{\partial w}{\partial z}=g\left(\alpha \Delta_{0} T+\beta \Delta_{0} s-(\alpha \Delta \gamma-\beta b) \cdot z\right), \\
& \frac{\partial u}{\partial x}+\frac{\partial w}{\partial z}=0
\end{aligned}
$$

Здесь мы при записи уравнения (8) сделали допущение, такое же, как в работе [22]:

$$
-\frac{1}{\rho_{\mathrm{e}}} \frac{\partial p^{\prime}}{\partial z}<<g(\alpha \Delta T+\beta \Delta s) \text {. }
$$

Из уравнения неразрывности (9) следует, что можно ввести функцию тока $\psi$ следующим образом:

$$
u=-\frac{\partial \psi}{\partial z}, \quad w=\frac{\partial \psi}{\partial x} .
$$

Сравнивая эту систему с системой уравнений тепловой конвекции сухого воздуха [42], замечаем, что они отличаются только лишь правой частью в уравнении (8). Поэтому решение системы уравнений (7) - (9) находится аналогично системе уравнений для сухого воздуха.

Из формулы (6) следует, что, если градиент массовой доли водяного пара будет приближаться к критическому значению, равному $b_{\text {cr }}=\alpha \Delta \gamma / \beta$, уровень выравнивания плотностей будет стремиться к бесконечности, произой- 
дет так называемое «взрывное» усиление конвекции. Данный факт является отличительной особенностью конвекции во влажной атмосфере по отношению к конвекции сухого воздуха.

Таким образом, мы видим, что критическим параметром для конвекшии является не абсолютное значение массовой доли водяного пара вблизи поверхности земли, а его вертикальный градиент.

Найдем значение критического градиента массовой доли водяного пара при условии, что $\gamma=0$

$$
\left(b_{\mathrm{ci}}\right)_{\max }=\frac{\alpha \Delta \gamma}{\beta}=10 \cdot 10^{-5} \mathrm{~m}^{-1}
$$

Для случая конвекции влажного ненасыщенного воздуха функция тока имеет вид

$$
\psi=\frac{\sqrt{g(\alpha \Delta \gamma-\beta b)}}{k} \sqrt{z\left(2 z_{\rho}-z\right)} \cos k x .
$$

Отсюда для проекций скорости получим выражения:

$$
\begin{aligned}
& u=\frac{\sqrt{g(\alpha \Delta \gamma-\beta b)}}{k} \frac{z_{\rho}-z}{\sqrt{z\left(2 z_{\rho}-z\right)}} \cos k x, \\
& w=\sqrt{g(\alpha \Delta \gamma-\beta b)} \cdot \sqrt{z\left(2 z_{\rho}-z\right)} \sin k x .
\end{aligned}
$$

Отсюда из условия $w=0$ для уровня конвекции влажного ненасыщенного воздуха получим выражение

$$
z_{w}=2 z_{\rho}=2 \frac{\alpha \Delta_{0} T+\beta \Delta_{0} s}{\alpha \Delta \gamma-\beta b} .
$$

Из (14) видно, что уровень конвекции зависит, как от перегрева и пересыщения в приземном слое, так и от вертикальных градиентов температуры и массовой доли водяного пара. Оценки параметров, входящих в формулу (14) показывают, что фактор перегрева $\Delta_{0} T$ сильнее влияет на развитие конвекции, чем фактор пересыщения $\Delta_{0} S$. Как видно из формулы (14) наиболее существенным фактором является градиент массовой доли водяного пара $b$. Если градиент массовой доли водяного пара приближается к критическому значению, то происходит «взрывное» усиление конвекции, т.е. уровень конвекции растет и стремится к бесконечности. По поводу «взрывного» роста следует заметить, что в нашей модели конвекции не учитывается вязкость воздуха. Поэтому, учет вязкости ограничит рост уровня конвекции.

Уровень максимальных скоростей найдем из условия: $d w / d z=0$. Взяв производную по переменной $z$ в выражении (13), получим 


$$
\frac{\partial w}{\partial z}=\tilde{N}_{\mathrm{BV}} \frac{z_{\rho}-z}{\sqrt{z\left(2 z_{\rho}-z\right)}} \sin k x=0,
$$

где величину

$$
\tilde{N}_{\mathrm{BV}}=\sqrt{g(\alpha \Delta \gamma-\beta b)}
$$

назовем частотой Брента - Вяйсяля колебаний влажного ненасыщенного воздуха. Из этой формулы видно, что при критическом градиенте массовой доли водяного пара частота Брента-Вяйсяля равна нулю, а значит, период колебаний стремится к бесконечности. Из формулы (15) следует, что уровень максимальных скоростей совпадает с уровнем выравнивания плотностей: $z_{w \max }=z_{\rho}$.

Подставляя выражение для величины $z_{\rho}$ в формулу (13), для максимальной скорости восходящих потоков влажного ненасыщенного воздуха получим равенство

$$
w_{\max }=\tilde{N}_{\mathrm{BV}} z_{\rho} \sin k x=\sqrt{\frac{g}{\alpha \Delta \gamma-\beta b}}\left(\alpha \Delta_{0} T+\beta \Delta_{0} s\right) \sin k x
$$

Запишем выражение для квадрата скорости восходящих потоков влажного ненасыщенного воздуха:

$$
w^{2}=\left[g(\alpha \Delta \gamma-\beta b) z\left(2 z_{\rho}-z\right)\right] \sin ^{2} k x .
$$

Отсюда следует, что, если $z_{\rho}=0$, т. е. плотности воздуха возмущенной и невозмущенной атмосферы у поверхности земли совпадают, то условием развития конвекции во влажной атмосфере является условие $b>b_{\text {er }}$. В частности, для сухой атмосферы отсюда следует известное условие развития конвекции: $\gamma>\gamma_{\mathrm{a}}$. Если же атмосфера влажная, то конвекция развивается даже при условии $\gamma<\gamma_{\mathrm{a}}$.

Тогда для квадрата скорости восходящих потоков получим выражение

$$
w=\sqrt{\beta g\left(b-b_{\mathrm{cr}}\right)} z \cdot \sin k x,
$$

т. е. скорость восходящих потоков будет расти с высотой по линейному закону и уровень конвекции не будет достигнут, даже если $\gamma<\gamma_{\mathrm{a}}$. Ясно, что полученный результат является идеализацией. В реальной атмосфере инверсионные слои и вязкость воздуха (напомним, что в нашей модели рассматривается уравнение движение идеальной жидкости) не дадут реализоваться указанному сценарию.

Выражение для амплитуды максимальной скорости восходящего потока во влажной атмосфере имеет вид

$$
w_{0}=\sqrt{\frac{g}{\alpha \Delta_{y}-\beta b}}\left(\alpha \Delta_{0} T+\beta \Delta_{0} s\right) .
$$


Таким образом, из полученного выражения (20) для амплитуды скорости восходящего потока видно, что вертикальная скорость зависит от неустойчивости атмосферы, градиента массовой доли водяного пара в окружающей атмосфере и перегрева у поверхности земли. Из формулы (20) также видно, что при критическом значении массовой доли водяного пара максимальная скорость стремится к бесконечности («взрывной рост»)

Значения параметров конвекции на уровне конденсации

Определим уровень конденсации $z_{\mathrm{c}}$ по формуле [21]:

$$
z_{\mathrm{c}}=\frac{T_{0}-\tau_{0}}{\gamma_{\mathrm{a}}-\gamma_{\tau}}=\frac{d_{0}}{\Delta \gamma_{\tau}}
$$

где $\tau_{0}-\quad$ температура точки росы у поверхности земли;

$d_{0}-\quad$ дефицит точки росы вблизи поверхности земли $(z=0)$;

градиент температуры точкиросы.

Как известно, формула (21) дает заниженные значения уровня конденсации, так как при ее выводе не учитывается вовлечение поднимающегося воздуха с окружающей средой. Для дальнейшего анализа имеет значение только лишь зависимость уровня конденсации от дефицита точки росы вблизи поверхности земли.

На уровне конденсации $z_{\mathrm{c}}$ выражение для скорости восходящих потоков будет иметь вид:

$$
w_{\mathrm{c}}^{2}=2 g\left(\alpha \Delta_{0} T+\beta \Delta_{0} s\right) \frac{d_{0}}{\Delta \gamma_{\tau}}\left(1-\frac{d_{0}}{2\left(\alpha \Delta_{0} T+\beta \Delta_{0} s\right)} \frac{\alpha \Delta \gamma-\beta b}{\Delta \gamma_{\tau}}\right) \sin ^{2} k x .
$$

Подставляя выражение (21) в формулу (2), получим формулу для функции перегрева на уровне конденсации

$$
\Delta_{\mathrm{c}} T=\Delta_{0} T-d_{0} \frac{\Delta \gamma}{\Delta \gamma_{\tau}}
$$

Для функции пересышения на уровне конденсации получим выражение

$$
\Delta_{\mathrm{c}} s=\Delta_{0} s+b \frac{d_{0}}{\Delta \gamma_{\tau}} .
$$

Из выражений (22) и (23) видно, что существуют критические значения дефицита точки росы вблизи поверхности земли, при которых соответственно равны нулю функция перегрева на уровне конденсации $\Delta_{\mathrm{c}} T$ и скорость восходящих потоков воздуха $w_{\mathrm{c}}$ (на уровне конденсации):

$$
\begin{aligned}
& \Delta_{\mathrm{c}} T=0:\left(d_{0}\right)_{\mathrm{cr} 1}=\Delta_{0} T \frac{\Delta \gamma_{\tau}}{\Delta \gamma}, \\
& w_{\mathrm{c}}=0:\left(d_{0}\right)_{\mathrm{cr} 2}=2\left(\alpha \Delta_{0} T+\beta \Delta_{0} s\right) \frac{\Delta_{\gamma \tau}^{\gamma}}{\alpha \Delta \gamma-\beta b} .
\end{aligned}
$$


Из формулы (26) следует, что влажность подоблачного слоя будет способствовать развитию облачной конвекции. Более того, из этой формулы следует, что при значении градиента массовой доли водяного пара, равного критическому значению $b_{\mathrm{cr}}=\frac{\alpha \Delta \gamma}{\beta}$, критическое значение дефицита точки росы вблизи поверхности земли $\left(d_{0}\right)_{\text {сг }}$ стремится к бесконечности, а это значит, что подоблачная конвекция будет всегда «пробивать» уровень конденсации, т. е. $w_{\mathrm{c}}>0$.

Полученные критерии определяют начальные условия облачной конвекции.

1. Если дефицит точки росы вблизи поверхности земли равен второму критическому значению $d_{0}=\left(d_{0}\right)_{\mathrm{c} 2}$, то на уровне конденсации перегрев будет отрицательным:

$$
\Delta_{\mathrm{c}} T=-\frac{(\alpha \Delta \gamma+\beta b) \Delta_{0} T+2 \beta \Delta \gamma \Delta_{0} s}{\alpha \Delta \gamma-\beta b}=-\left(\Delta_{\mathrm{c}} T\right)_{\max }<0,
$$

а скорость восходящего потока на этом же уровне будет равна нулю $(w c=0)$. Следовательно, в этом случае подоблачный слой не будет способствовать развитию облачной конвекции. То есть атмосфера при таком дефиците точки росы вблизи поверхности земли недостаточно влажная, и подоблачный слой не будет пробиваться восходящим воздухом (рис. 1, график 1).

Формально принимая $\beta=0$, получим, что для «сухой подоблачной атмосферы» функция перегрева на уровне конденсации будет равна $\Delta_{\mathrm{c}} T=-\Delta_{0} T<0$. Данный результат известен из адиабатической модели конвекции сухого воздуха [21].

2. Если дефицит точки росы вблизи поверхности земли $\left(d_{0}\right)_{\mathrm{cr} 1}<d_{0}<\left(d_{0}\right)_{\mathrm{cr} 2}$, то перегрев на уровне конденсации будет отрицательным, но по модулю меньше максимального значения $-\left(\Delta_{\mathrm{c}} T\right)_{\max }<\Delta_{\mathrm{c}} T<0$, а скорость восходящего потока на этом же уровне положительна. В этом случае уровень конденсации будет пробиваться восходящей подоблачной струёй, что способствует развитию облачной конвекции (рис. 1, график 2).

3. Если дефицит точки росы вблизи поверхности земли равен первому критическому значению $d_{0}<\left(d_{0}\right)_{\mathrm{er} 1}$, то на уровне конденсации перегрев равен нулю $\Delta_{\mathrm{c}} T=0$, а скорость восходящего потока будет равна $w_{\mathrm{c}}\left(z_{\mathrm{t}}\right)$ (меньше максимального значения), т.е. равна значению на уровне $z_{\mathrm{t}}$ выравнивания температур:

$w_{\mathrm{c}}^{2}=g(\alpha \Delta \gamma-\beta b) z_{\mathrm{t}}\left(2 z_{\rho}-z_{\mathrm{t}}\right) \sin ^{2} k x$ 
Заметим, что в этом случае уровень конденсации совпадает с уровнем выравнивания температур: $z_{\mathrm{c}}=z_{\mathrm{t}}$. Аналогично, функция пересыщения примет значение, равное значению на этом же уровне $z_{\mathrm{t}}$ :

$$
\Delta_{\mathrm{c}} s=\Delta_{0} s+b \frac{\Delta_{0} T}{\Delta \gamma}=\Delta_{0} s+b z_{\mathrm{t}}
$$

Следовательно, в этом случае будут существовать условия для развития облачной конвекции (рис. 1, график 3 ).

4. Если дефицит точки росы вблизи поверхности земли меньше первого критического значения дефицита $d_{0}<\left(d_{0}\right)_{\mathrm{cr} 1}$, то перегрев на уровне конденсации положителен $\Delta_{0} T>0$, а скорость восходящего потока будет положительной, но меньше максимального значения, что также может способствовать развитию облачной конвекции (рис. 1, график 4).

$$
\begin{array}{cc}
d_{0}=\left(d_{0}\right)_{\mathrm{cr} 2}, \Delta_{\mathrm{c}} T=-\left(\Delta_{0} T\right)_{\max }, & d_{0}<\left(d_{0}\right)_{\mathrm{cr} 2,},-\left(\Delta_{0} T\right)_{\max }<\Delta_{\mathrm{c}} T<0, \\
w_{\max }=0 & w_{\mathrm{c}}>0, \quad \partial w / \partial z<0
\end{array}
$$

Из выражения (22) видно, что, если градиент массовой доли водяного пара в окружающей атмосфере принимает критическое значение $b_{\text {сг }}$, то скорость восходящих потоков на уровне конденсации при любом значении дефицита точки росы вблизи поверхности земли положительна и равна:

$$
w_{\mathrm{c}}^{2}=2 g\left(\alpha \Delta_{0} T+\beta \Delta_{0} s\right) \frac{d_{0}}{\Delta \gamma_{\tau}} .
$$

Итак, учет влажности подоблачного слоя приводит не только к количественным изменениям критериев, определяющих параметры облачной конвекции на уровне конденсации, но и качественным изменениям. Мы видим, что существенное влияние на параметры облачной конвекции на уровне конденсации имеет не столько значение абсолютной влажности подоблачного слоя, сколько градиент массовой водяного пара в подоблачном слое

Таким образом, полученное в работеаналитическое выражение для двумерной модели конвекции позволило провести анализ влияния характеристик приземного слоя атмосферына развитие облачной конвекции.

\section{Результаты исследования и их обсуждение}

В работе [43] получено аналитическое решение двумерной модели конвекции влажного насыщенного воздуха. Приведем основные положения и допущения, на которых было основано аналитическое решение задачи в работе [43]. 


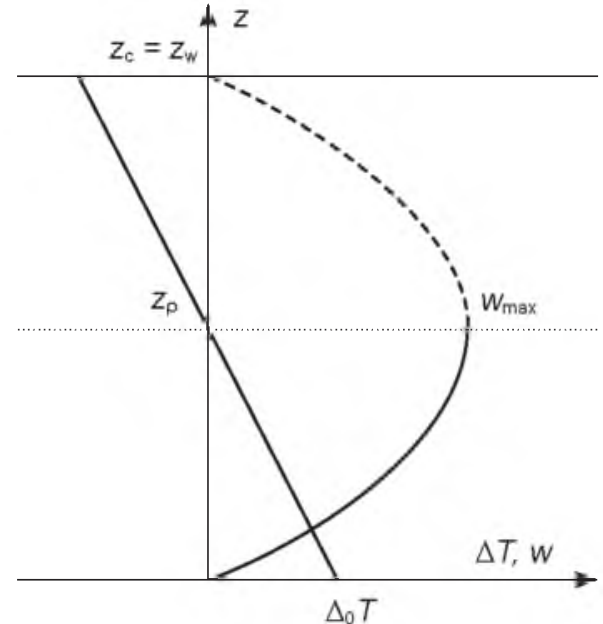

График 1

$d_{0}<\left(d_{0}\right)_{\mathrm{crl}}, \Delta_{\mathrm{c}} T=0, d w / d z=0$

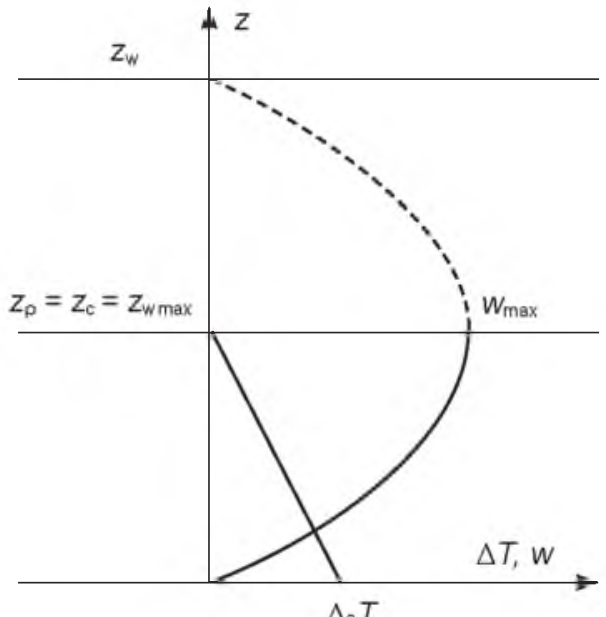

График 3

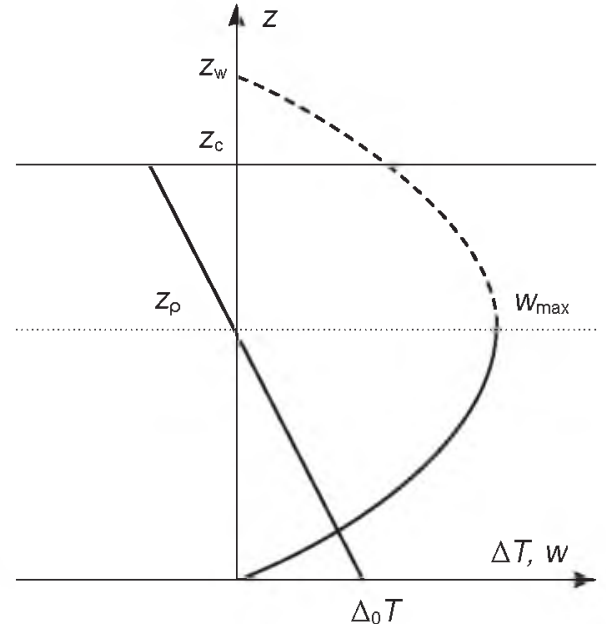

Графикк 2

$d_{0}<\left(d_{0}\right)_{\mathrm{cr}}, \Delta_{\mathrm{c}} T>0, d w / d z>0$.

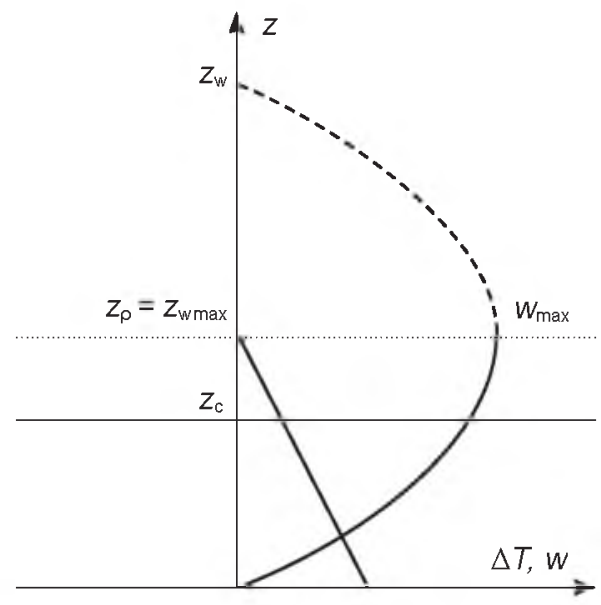

График 4

Рис. 1.

Распределение скорости восходящих потоков и функции перегрева с высотой в зависимости от различных значений дефицита точки росы вблизи поверхности земли.

Fig. 1. The distribution of the ascending flow velocity and the overheating function with height depending on various values of the dew point deficit near the earth's surface. 
Градиент температуры влажного насыщенного воздуха представлен нами в виде [43]

$$
\begin{aligned}
& \gamma_{\mathrm{ma}}=-\frac{\mathrm{d} T_{\mathrm{i}}}{\mathrm{d} z}=\gamma_{\mathrm{a}}+\frac{L}{c_{p}} \frac{\mathrm{d} s_{\mathrm{m}}}{\mathrm{d} z}=\gamma_{\mathrm{mac}}+\varepsilon\left(z-z_{\mathrm{c}}\right), \\
& \gamma_{\mathrm{mac}}=\gamma_{\mathrm{a}}+\left.\frac{L}{c_{p}} \frac{\mathrm{d} s_{\mathrm{m}}}{\mathrm{d} z}\right|_{z=z_{\mathrm{c}}}
\end{aligned}
$$

- влажноадиабатический градиент температуры на уровне конденсации - известная функция температуры и давления $\left(\frac{\mathrm{d} s_{\mathrm{m}}}{\mathrm{d} z}<0\right)[21]$

$L-\quad$ удельная теплота конденсации;

$c_{p}-\quad$ удельная теплоемкость при постоянном давлении; $s_{\mathrm{m}}$ массовая доля насыщенного водяного пара;

$\varepsilon=\frac{L}{c_{p}}|K| \approx 3 \cdot 10^{-7 \circ^{\circ}} \mathrm{C} / \mathrm{m}^{2}-$ константа;

$K=\left.\frac{\mathrm{d}^{2} s_{\mathrm{m}}}{\mathrm{d} z^{2}}\right|_{z=z_{c}}-$ некоторая функция от величины $s_{\mathrm{mc}}-$ массовой доли водяного пара на уровне конденсации, также принимается постоянной.

Функшия перегрева запишется в виде:

$$
\Delta T(z)=\Delta_{\mathrm{c}} T+\Delta \gamma_{\operatorname{mac}}\left(z-z_{\mathrm{c}}\right)-\frac{\varepsilon}{2}\left(z-z_{\mathrm{c}}\right)^{2},
$$

где $\quad \Delta_{\mathrm{c}} T-\quad$ значение функции перегрева на уровне конденсации;

$\Delta \gamma_{\operatorname{mac}}=\gamma-\gamma_{\text {mac }}-$ разность градиентов температуры воздуха невозмущенной атмосферы и поднимающейся частицы на уровне конденсации. На аэрологической диаграмме $\Delta \gamma_{\text {mac }}$ определяет угол между кривой состояния и кривой стратификации на уровне конденсации.

Для функции пересыщения получено выражение:

$$
\begin{aligned}
& \Delta s(z)=\Delta_{\mathrm{c}} s+\left(b+\left.\frac{\mathrm{d} s_{m}}{\mathrm{~d} z}\right|_{z=z_{\mathrm{c}}}\right)\left(z-z_{\mathrm{c}}\right)+\frac{K}{2}\left(z-z_{\mathrm{c}}\right)^{2}, \\
& \text { где } \quad \Delta_{\mathrm{c}} s=s_{\mathrm{mc}}-s_{\mathrm{ec}}-\text { пересыщение на уровне конденсации }
\end{aligned}
$$

Формула для плотности воздушной частицы запишется в виде

$$
\rho_{\mathrm{i}}=\rho_{\mathrm{e}}\left[1-\alpha_{0}-\alpha_{1}\left(z-z_{\mathrm{c}}\right)+\alpha_{2}\left(z-z_{\mathrm{c}}\right)^{2}\right],
$$


где

$$
\alpha_{0}=\alpha \Delta_{\mathrm{c}} T+\beta \Delta_{\mathrm{c}} s
$$$$
\alpha_{1}=\alpha \Delta \gamma_{\operatorname{mac}}+\beta\left(b+\left.\frac{\mathrm{d} s_{m}}{\mathrm{~d} z}\right|_{z=z_{\mathrm{c}}}\right), \alpha_{2}=\frac{1}{2}(\alpha \varepsilon-\beta K) .
$$

Уровень выравнивания плотностей воздуха в облачной воздушной частице и окружающей атмосфере

$$
z_{\rho}-z_{\mathrm{c}}=\frac{\alpha_{1}+\sqrt{\alpha_{1}^{2}+2(\alpha \varepsilon-\beta K)\left(\alpha \Delta_{\mathrm{c}} T+\beta \Delta_{\mathrm{c}} s\right)}}{\alpha \varepsilon-\beta K} .
$$

Уровень выравнивания температур определяется выражением:

$$
z_{\mathrm{t}}-z_{\mathrm{c}}=\frac{\Delta \gamma_{\mathrm{mac}}+\sqrt{\left(\Delta \gamma_{\mathrm{mac}}\right)^{2}+2 \varepsilon \Delta_{\mathrm{c}} T}}{\varepsilon}
$$

Тепловая конвекция влажного насыщенного воздуха описывается системой уравнений

$$
\begin{aligned}
& u \frac{\partial u}{\partial x}+w \frac{\partial u}{\partial z}=-\frac{1}{\rho_{\mathrm{e}}} \frac{\partial p^{\prime}}{\partial x}, \\
& u \frac{\partial w}{\partial x}+w \frac{\partial w}{\partial z}=g(\alpha \Delta T+\beta \Delta s), \\
& \Delta T(z)=\Delta_{\mathrm{c}} T+\Delta \gamma_{\operatorname{mac}}\left(z-z_{\mathrm{c}}\right)-\frac{\varepsilon}{2}\left(z-z_{\mathrm{c}}\right)^{2}, \\
& \Delta s(z)=\Delta_{\mathrm{c}} s+\left(b+\left.\frac{\mathrm{d} s_{m}}{\mathrm{~d} z}\right|_{z=z_{\mathrm{c}}}\right)\left(z-z_{\mathrm{c}}\right)+\frac{K}{2}\left(z-z_{\mathrm{c}}\right)^{2}, \\
& \frac{\partial u}{\partial x}+\frac{\partial w}{\partial z}=0 .
\end{aligned}
$$

Решая эту систему, для функции тока получим выражение

$$
\psi=\frac{\tilde{N}_{\text {mac }}}{k} \sqrt{2\left(z-z_{\mathrm{c}}\right)\left[\tilde{z}_{1}+\frac{\left(z-z_{\mathrm{c}}\right)}{2}-\frac{k_{1}}{3}\left(z-z_{\mathrm{c}}\right)^{2}\right]} \cos k x,
$$


где

$$
\tilde{N}_{\text {mac }}=\sqrt{g\left[\alpha \Delta \gamma_{\text {mac }}+\beta\left(b+\left.\frac{\mathrm{d} s_{m}}{\mathrm{~d} z}\right|_{z=z_{\mathrm{c}}}\right)\right]}
$$

- частота Брента - Вяйсяля для влажного насыщенного воздуха;

$$
\begin{aligned}
& k_{1}=\frac{\alpha \varepsilon-\beta K}{2\left[\alpha \Delta \gamma_{\text {mac }}+\beta\left(b+\left.\frac{\mathrm{d} s_{m}}{\mathrm{~d} z}\right|_{z=z_{\mathrm{c}}}\right)\right]}, \\
& \tilde{z}_{1}=\frac{\alpha \Delta_{\mathrm{c}} T+\beta \Delta_{\mathrm{c}} s}{\alpha \Delta \gamma_{\text {mac }}+\beta\left(b+\left.\frac{\mathrm{d} s_{m}}{\mathrm{~d} z}\right|_{z=z_{\mathrm{c}}}\right)},
\end{aligned}
$$

Для вертикальной скорости получим выражения:

$$
w=\sqrt{w_{\mathrm{c}}^{2}+2 \tilde{N}_{\text {mac }}^{2}\left(z-z_{\mathrm{c}}\right)\left[\tilde{z}_{1}+\frac{\left(z-z_{\mathrm{c}}\right)}{2}-\frac{k_{1}}{3}\left(z-z_{\mathrm{c}}\right)^{2}\right] \cdot \sin ^{2} k x}
$$

Толщина конвективной ячейки («облака») находится из условия $w=0$ и для случая, когда скорость на уровне конденсации равна нулю $\left(w_{\mathrm{c}}=0\right)$, равна

$$
z_{w}-z_{\mathrm{c}}=\frac{3}{4 k_{1}}\left(1+\sqrt{1+\frac{16}{3} k_{1} \tilde{z}_{1}}\right)
$$

Так как согласно формуле (32) $\frac{\mathrm{d} s_{\mathrm{m}}}{\mathrm{d} z}<0$, то из формул (47) видно, что и в случае облачной конвекции существует критическое значение градиента массовой доли водяного пара окружающей среды, которое способствует развитию облачной конвекции:

$$
b_{\mathrm{cr}}=\left(-\left.\frac{\mathrm{d} s_{m}}{\mathrm{~d} z}\right|_{z=z_{\mathrm{c}}}\right)-\frac{\alpha}{\beta} \Delta \gamma_{\mathrm{mac}}
$$

Более того, из выражений (45) и (47) видно, что когда $\Delta \gamma_{\text {mac }}$ $<0$, т. е. атмосфера влажно устойчива и согласно традиционным представлениям конвекция не возможна, то при значениях градиента массовой доли водяного пара, больших критического значения, и в этом случае конвекция возможна.

На рисунке 2 функшия тока для подоблачной конвекции рассчитывалась по формуле (11), а для облачной конвекции по формуле (43). 


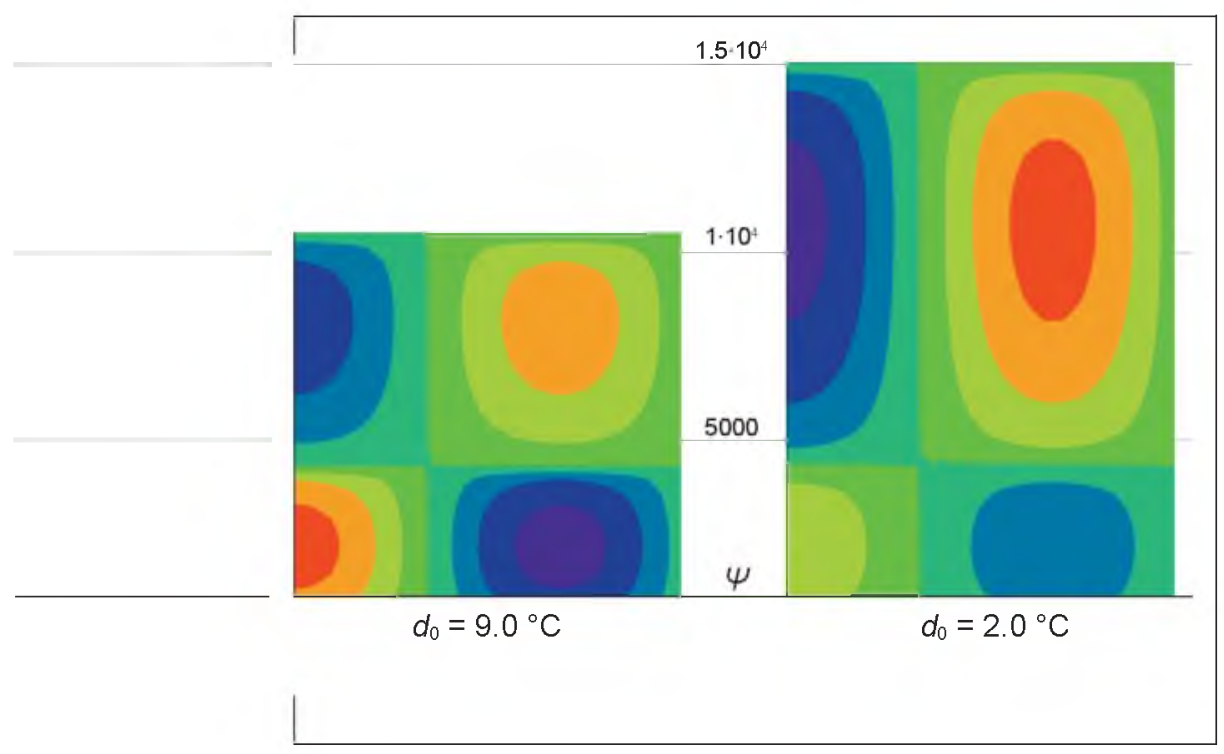

Рис. 2.

\author{
Взаимодействие подоблачной и облачной конвекции. \\ На рисунке по осям x и z отложены метры. \\ Fig. 2. Interaction of under-cloud and cloud convection. \\ In the figure along the axes $x$ and $z$ laid off meters
}

Из рисунка видно, что увеличение влажности в приземном слое приводит к развитию облачной конвекции.

Таким образом, из полученных выражений для параметров облачной конвекции видно, что она зависит от значений функции перегрева $\Delta_{\mathrm{c}} T$ и скорости восходящих потоков $w_{c}$ на уровне конденсации, которые сами зависят от характеристик приземного слоя атмосферы.

\title{
Выводы
}

На основании полученных в работах $[42,43]$ аналитических решений уравнений конвекции сухого и влажного насыщенного воздуха, а также аналитического решения конвекции влажного ненасыщенного воздуха, показано влияние на развитие облачной конвекции параметров приземного слоя атмосферы. Получены аналитические выражения для дефицитов точки росы в приземном слое, определяющие значения функции перегрева и скорости восходящих потоков на уровне конденсации. Показано, что на развитие подоблачной конвекции существенное влияние оказывает значение массовой доли водяного пара. Установлено, что при значениях градиента массовой доли водяного пара, приближающихся к критическому значению, наблюдается «взрывной» рост конвективных ячеек в подоблачном слое. Также показа- 
но, что существует критическое значение градиента массовой доли водяного, способствующее развитию облачной конвекции.

Таким образом, основной результат, следуемый из предложенной теоретической модели, о влиянии влажности приземного слоя атмосферы на развитие конвекции, находится в согласии с многочисленными экспериментальными и теоретическими исследованиями, обзор которых был проведен во введении. Отличительной особенностью нашей модели является то, что, во-первых, мы говорим не просто об абсолютном значении влажности приземного слоя, а о критическом значении градиента массовой доли водяного пара. Кроме того, для анализа мы находили решение двумерной нелинейной модели конвекции. В качестве недостатка нашей модели отметим ее стационарность и отсутствие вязкости.

\section{Библиографический список}

1. Тихонов А.Н., Самарский А.А. Уравнения математической физики. М.: Изд-во МГУ. 6-е издание. 1999. 799 с.

2. Alekseev V.V., Gusev A.M. Free convection in geophysical processes. Sov. Phys. Uspekhi 1983, 26, 906-922.

3. Arakawa A. The cumulus parameterization problem: Past, present and future, J. Climate, 17, 2493-2525, 2004.

4. Bellon G., and Stevens B., 2005: On bulk models of shallow cumulusconvection. J. Atmos. Sci., 62, 3286-3302.

5. Bennett L.J., Browing K.A., Blyth A.M., Parker D.J., and Clark P.A. 2006: A review of the initiation of precipitating convection in the United Kingdom. Q.J.R. Meteorol. Soc., 132, 1001-1020.

6. Betts A.K., Ball J.H., Beljaars A. C.M., Miller M.J., and Viterbo P.A.: The land surface-atmosphere interaction: A review based on observational and global modeling perspectives, J. Geophys. Res.Atmos., 101, 7209-7225, 1996.

7. Bluestein H.B. Severe Convective Storms and Tornadoes. Chichester, UK: Springer, 2013. $481 \mathrm{p}$

8. Bony S., and Dufresne J.L., 2005: Marine boundary layer clouds at the heart of tropical cloud feedback uncertainties in climate models Geophys. Res. Let., 32, L20806.

9. Bretherton C.S., and Park S., 2008: A new bulk shallowcumulusmodel and implications for penetrative entrainment feedbackon updraft buoyancy. J. Atmos. Sci., 65, 2174-2193.

10. Brooks H.E., Doswell C.A., and Wicker L.J., 1993: STORM-TIPE A forecastingexperiment using a three-dimensional cloud model Wea. Forecasting, 8, 352-362.

11. Browning K., Blyth A., Clark P., Corsmeier U., Morcrette C, Agner J., Bamber D., Barthlott C., Bennett L., Beswick K., Bitter M., Bozier K.,. Brooks B,. Collier C, Cook C., Davies F., Deny B., Engelhardt M., Feuerle T., Forbes R., Gaffard C., Gray M., Hanken R., Hewison T., Huckle R., Kalthoff N., Khodayar S., Kholer M., Kraut S., Kunz 
M., Ladd D., Lenfant J., Marsham J., McGregor J., Nicol J., Norton E., Parker D., Perry F., Ramatschi M., Roberts H., Russel A., Schulz H., Slack E., Vauhan G., Waight J., Watson R., Webb A., Wieser A., and Zinz K., 2007: The Convective Storm Initiation Project. Bull. Am. Meteorol. Soc., 1-17.

12. Crook N. A. Sensitivity of Moist Convection Forced by Boundary Layer Processes to Low-Level Thermodynamic Fields. Monthly Weather Review. Volume 124, 1996, p. 1767-1785.

13. Deardorff J.W., 1979: Prediction of convective mixed-layer entrainment forrealistic capping inversion structure. J. Atmos. Sci., $36,424-436$

\section{References}

1. Tikhonov A.N., Samara A.A. Equations of mathematical physics M.: MGU Publishing House. 6th edition. 1999. 799 p.

2. Alekseev V.V., Gusev A.M. Free convection in geophysical processes. Sov. Phys. Uspekhi 1983, 26, 906-922.

3. Arakawa A. The cumulus parameterization problem: Past, present, and future, J. Climate, 17, 2493-2525, 2004.

4. Bellon G., and Stevens B., 2005: On bulk models of shallow cumulusconvection. J. Atmos. Sci., 62, 3286-3302.

5. Bennett L.J., Browing K.A., Blyth A. M., Parker D.J., and Clark P.A., 2006: A reviewof the initiation of precipitating convection in the United Kingdom. Q.J.R. Meteorol. Soc., 132, 1001-1020.

6. Betts A.K., Ball J.H., Beljaars A.C.M., Miller M.J., and Viterbo P.A. The land surface-atmosphere interaction: A review based on observational and global modeling perspectives, J. Geophys. Res.Atmos., 101, 7209-7225, 1996.

7. Bluestein H.B. Severe Convective Storms and Tornadoes. Chichester, UK: Springer, 2013. $481 \mathrm{p}$.

8. Bony S., and Dufresne J.L., 2005: Marine boundary layer clouds at the heart of tropical cloud feedback uncertainties in climate models Geophys. Res. Let., 32, L20806.

9. Bretherton C.S., and Park S., 2008: A new bulk shallow-cumulusmodel and implications for penetrative entrainment feedbackon updraft buoyancy. J. Atmos. Sci., 65, 2174-2193.

10. Brooks H.E., Doswell C.A., and Wicker L.J., 1993: STORM-TIPE A forecastingexperiment using a three-dimensional cloud model. Wea. Forecasting, 8, 352-362.

11. Browning K., Blyth A., Clark P., Corsmeier U., Morcrette C, Agner J., Bamber D., Barthlott C., Bennett L., Beswick K., Bitter M., Bozier K., Brooks B,. Collier C, Cook C., Davies F., Deny B., Engelhardt M., Feuerle T., Forbes R., Gaffard C., Gray M., Hanken R., Hewison T., Huckle R., Kalthoff N., Khodayar S., Kholer M., Kraut S., Kunz M., Ladd D., Lenfant J., Marsham J., McGregor J., Nicol J., Norton E., Parker D., Perry F., Ramatschi M., Roberts H., Russel A., Schulz H., Slack E., Vauhan G., Waight J., Watson R., Webb A., Wieser A., and Zinz K., 2007: The Convective Storm Initiation Project. Bull. Am. Meteorol. Soc., 1-17. 
12. Crook N. A. Sensitivity of Moist Convection Forced by Boundary Layer Processes to Low-Level Thermodynamic Fields. Monthly Weather Review. Volume 124, 1996, p. 1767-1785.

13. Deardorff J.W., 1979: Prediction of convective mixed-layer entrainment forrealistic capping inversion structure. J. Atmos. Sci., 36 $424-436$

\section{O6 авторах}

Данилова Нина Евгеньевна, аспиранта кафедры общей и теоретической физики Северо-Кавказского федерального университета. Тел 8-918-769-22-07 E-mail: ninochka841@mail.ru

Семенова Юлия Александровна, аспиранта кафедры общей и теоретической физики Северо-Кавказского федерального университета. Тел. 8-918-753-65-56

E-mail: brilliance_wave@mail.ru

Смерек Юлия Леонтьевна, кандидат физико-математических наук, доцент кафедры общей и теоретической ффизики Северо-Кавказского федерального университета. Тел. 8(918)758-71-29 E-mail smerek@mail.ru, Scopus ID: 6503952686

ResearcherlD is: Q-5159-2018

Закинян Роберт Гургенович, доктор физико-математических наук, профессор кафедры общей и теоретической физики Северо-Кавказского федерального университета

Тел. 8(918)77-88-675 E-mail: zakinyan@mail.ru

\section{About the authors}

Danilova Nina E., Postgraduate Student, Department of General and Theoretical Physics, North-Caucasian Federal University, E-mail ninochka841@mail.ru

Semenova YuliaAlexandrovna, Postgraduate Student, Department of General and Theoretical Physics, North-Caucasian Federal University, Email: brilliance_wave@mail.ru

Smerek Yulia Leontevna, Candidate of Physical and Mathematical Sciences, Associate Professor, Chair of General and Theoretical Physics, North Caucasus Federal University

Phone 8 (918) 758-71-29, E-mail: smerek@mail.ru

Scopus ID: 6503952686

ResearcherID is: Q-5159-2018

Zakinyan Robert Gurgenovich, The doctor of physical and mathematical sciences, Professor of the Department of General and Theoretical Physics IMNS NCFU

Telephone 8(918) 77-88-675 E-mail: zakinyan@mail.ru Scopus ID 7801621547 